\title{
Impact of Artificial Intelligence Integration on Surgical Outcome
}

\section{Dr. Muhammad Umar Younis}

Mediclinic City Hospital Dubai, UAE.

Correspondence to: Dr. Muhammad Umar Younis, Email: umarooo@hotmail.com, ORCiD: 0000-0001-6366-6991

\section{ABSTRACT}

Artificial Intelligence $(\mathrm{Al})$ is improving surgical practice with the technical advancement in imagery, navigation and robotic systems. This review is aimed at assessing the role of $\mathrm{Al}$ in surgical decision making during preoperative, intraoperative, and postoperative periods. Moreover, current trends pervading these aspects are scrutinized to emphasize need of evidence-based protocols in this regard. The search strategy involving key terms pertaining to the concepts was utilized. In order to reach maximum sensitivity, a combination of the terms such as "Artificial intelligence", "robotic surgery", "surgical decision making", "machine learning", "deep learning" and "Al" were considered. Only articles that specifically discussed the role of Al in surgical decision making were included. Fifty-five studies were retrieved with exclusion of forty-six of them according to inclusion criteria. Thus, nine studies were scrutinized in the final review and arranged in tabulated form to aid in data review and analysis. The advancement of Al has turned modern medicine into a more effective and efficient practice to manage both acute and chronic illnesses. Important advances from anatomical classifications through image guidance in pre-operative preparation to integration of data post discharge after surgery are minimizing the morbidity associated with a surgical procedure making the entire experience much seamless.

Keywords: Artificial Intelligence, Robotic Surgery, Surgical Decision Making, Machine Learning, Deep Learning.

This is an Open Access article distributed under the terms of the Creative Commons Attribution Non-Commercial License (http:// creative commons. org/licenses/by-nc/4.0) which permits unrestricted non-commercial use, distribution, and reproduction in any medium, provided the original work is properly cited.

\section{INTRODUCTION}

Artificial Intelligence $(\mathrm{Al})$ is a domain that develops smart algorithms and devices.' Surgeons offer complex plans when offering an operation, addressing risk factors, managing side effects, optimizing resource utilization, and performing a procedure. The single most abundant source of avoidable damage incurred by surgical patients is via diagnostic and assessment failures. ${ }^{2}$ Surgeons admit that assessment lapses are the most frequent cause of errors encountered in frequent practice. Hypothetical deductive reasoning and human intuition govern surgical decision-making by default and are extremely subjective and inadequate to correct such damages. ${ }^{3}$

Though clinical decision-making poses a significant challenge for surgeons, there is a relative lack of research examining this complex process and the strategies required for its improvement. Advances in surgery have had a major effect on the treatment of surgical illnesses, significantly improving the quality of life and expanding the limits of human survival continuously. Continuous technical advancements in evaluation, imaging, and surgical instrumentation are a part of this evolution. Meticulous surgical planning and preparations are made possible by using radiological J Dow Univ Health Sci 2021, Vol. 15 (2) : 103-109 advancements like ultrasonography, computed tomography scan (CT scan), and magnetic resonance imaging (MRI) both preoperatively as well as during surgery. ${ }^{4}$ Surgical trauma is being minimized by the improvement in minimally invasive surgery techniques, which now is frequently assisted with surgical robots; ${ }^{5}$ post-operative treatment is now strengthened by utilization of advanced sensors which can be worn or implanted in order to facilitate prompt discharge from hospital facility, to aid recovery from surgery and to immediately identify post-operative complications. ${ }^{6}$ The aim of this review was to study the applications of Al in pre-operative, intra-operative, as well as postoperative care. Initially, Al implementation in preoperative planning is discussed followed by $\mathrm{Al}$ approaches for intraoperative care. Technically, this analysis puts heavy emphasis on strategies focused on machine learning.

\section{METHODS}

This study utilized the PRISMA (Preferred Reporting Items for Systematic Reviews and Meta-Analyses) guidelines to make the basis of selection criteria using the PICO (P - Populations/People/Patient/Problem, I Intervention(s), C - Comparison, O - Outcome) 
worksheet as shown in table 1. PRISMA protocol was utilized to ensure a standardized approach to the development of this review. ${ }^{7}$ The PRISMA protocol is an essential checklist structured for reporting of systematic reviews and meta-analyses. (Fig.1)

Electronic databases PubMed, Elsevier, ResearchGate, Cochrane, and Web of Science were evaluated. The search strategy involved the key terms pertaining to the concepts and a combination of the terms "Artificial intelligence", "robotic surgery", "surgical decision making", "machine learning", "deep learning" and "Al" were considered in order to increase sensitivity of search. Relevant studies meeting the criteria were selected after reviewing the abstract of the study. The author further analyzed the references of the ascertained studies to evaluate for any supplementary papers.

Randomized controlled trials, case-control studies, and cohort studies fulfilling the following criteria were included: (1) Published in English language, (2) studies from the last 5 years ( 3 ) studies conducted on Humans only, and (4) novel or meritorious contribution to existing literature. If different studies were published by the same centre, only the most up to date was selected. Studies omitted from this review included: (1) studies with more than 5 years of publication (unless publication has extreme relevance up to this day) (2) Non relevant articles by abstract and content and (3) Case reports, editorials, and letters.

Studies not in the public domain were not included and those generated by the search were assessed for relevance. Potentially relevant papers were retrieved in full and evaluated by the author to minimize the risk of developing bias to the results reviewed. The complete text of the studies was thoroughly analyzed in order to assess the purpose and caliber of the study. Bias risk was evaluated using the Newcastle-Ottawa Quality Assessment Scale criteria.

\section{RESULTS}

A total of fifty-five studies were retrieved from the initial search. Abstracts were re-evaluated and forty-six of them were excluded due to being unrelated to the subject matter or not meeting the inclusion criteria. Thus, nine studies constituted the final review (Table 2) which were arranged in tabulated form.

In a study by Bagnall et al, ${ }^{8}$ the authors evaluated six traditional risk models in patients undergoing colorectal surgery at a single institution and concluded that these models were inefficient and overestimated mortality and morbidity. They, therefore, recommen- ded that new models were required to assess postoperative mortality rates. Adhikari et al. ${ }^{9}$ reported an improved predictive model using data from dynamic intraoperative time series variables to enrich a preoperative risk stratification system in order to correctly estimate acute kidney injury (AKI) in patients undergoing surgery. Their model shows a definite improvement in AKI prediction with this machine learning approach. Bertsimas et $\mathrm{al}^{10}$ devised an application "Predictive Optimal Trees in Emergency Surgery Risk"(POTTER) and compared its performance with various parameters. The author stated that POTTER might prove useful as a tool for bedside preoperative counseling of emergency surgery patients and families. Bihorac et al." devised a novel machine learning risk algorithm model (MySurgeryRisk) with a promising ability to determine complications and deathusing preoperative data. The study utilized the data involving 285 different variables to identify eight postoperative complications and estimated mortality at 1, 3, 6, 12months. A study by Brennan et al, $^{12}$ further lends credence to the MySurgeryRisk model confirming its feasibility and acceptance by the physician community. A number of studies reported similarly that the integration of Al during preoperative, intraoperative and post-operative periods accurately predicts mortality and morbidity of admitted patients. ${ }^{13-15}$

Stacey et al. ${ }^{16}$ carried out a review that determined with sufficient proof that individuals exposed to decision aids in the form of written, videos or web based tools using $\mathrm{Al}$, considered that they felt more positive and confident, more knowledgeable and assumed a significant dominant part in their treatment decisions. All these studies lend credence to the positive implications $\mathrm{Al}$ has had in the domain of surgical practice.

\section{DISCUSSION}

Al points to the development of computerized systems that tend to imitate important human behavioral functions including problem solving and rational investigation. A computer program that uses basic decision-tree functions will imitate human intelligence in the broadest sense. Al, however, typically applies to algorithms that are able to learn with significant autonomy from raw data, as is seen in the context of deep learning and machine learning. Al solutions when fed with continuous data streams, tend to resolve the shortcomings posed by conventional approaches to facilitate surgical decisions made through treatment adjuncts. $^{17}$ 

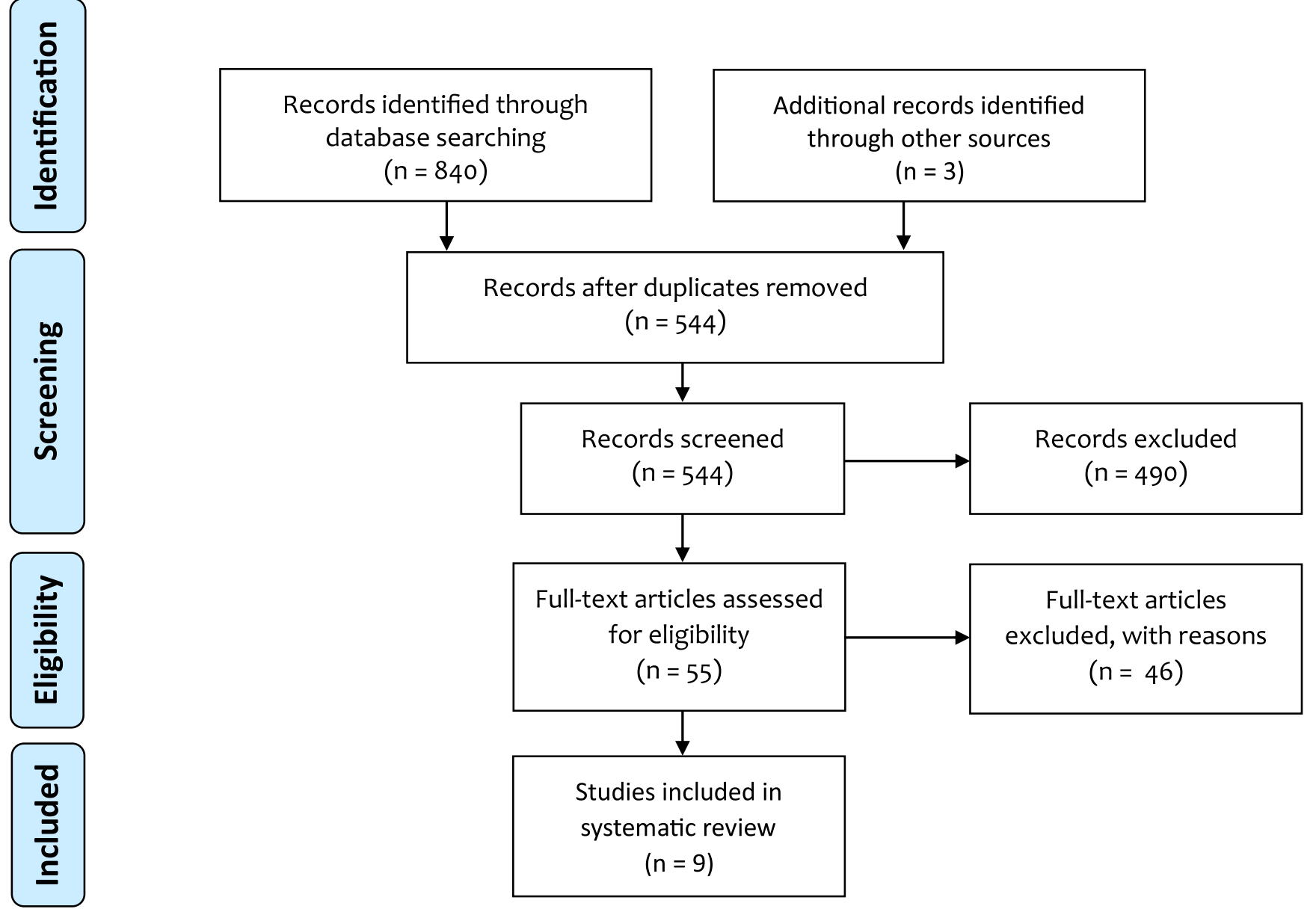

Figure 1: PRISMA flow diagram of study selection

Table 1: PICO (Patients, Intervention, Comparison, Outcome) criteria

\begin{tabular}{|c|c|}
\hline Patients & In patients undergoing surgery \\
\hline Intervention & Is Artificial intelligence \\
\hline Comparison & nparison with conventional techniques \\
\hline Effective in dec & easing morbidity and improving clinical outcome \\
\hline $\begin{array}{l}\text { The application of Al has advanced rapidly in the last } \\
\text { decade, and applied to medical data, and is highly } \\
\text { successful in analysis and modeling. }{ }^{15} \text { The explanation } \\
\text { behind this is the immense and rapid growth in the } \\
\text { ability of networked and mobile computing systems to } \\
\text { store and hold massive quantities of data. Jordan et al. } \\
\text { stated that, in the } 21 \text { st century, machine learning is } \\
\text { expected to be one of the most revolutionary } \\
\text { technologies. }{ }^{18} \text { This phenomenon will become more } \\
\text { and more prominent in the future, depending on the } \\
\text { technological growth. Al is broadly described as a } \\
\text { science comprising of algorithms, which gives various } \\
\text { systems the capacity of comprehension and } \\
\text { performance of cognitive functions such as deciphering } \\
\text { problems, perception of objects and words, and } \\
\text { decision-making. }\end{array}$ & $\begin{array}{l}\text { In surgery and healthcare, Al has evolved from simple } \\
\text { computer programs to complex clinical scenario } \\
\text { analysis tools enabling its integration into nearly every } \\
\text { clinical and organizational area. }{ }^{20} \text { Radiological } \\
\text { investigation was at the forefront of this transition } \\
\text { along with the use of augmented reality tools and } \\
\text { surgical robots in multiple branches of surgery. }{ }^{5} \text { They } \\
\text { were followed by other image-based specialties and, } \\
\text { more recently, by virtually all fields of surgery. The } \\
\text { "computer-aided diagnostic" systems have been } \\
\text { extended to include online assistants and robotic } \\
\text { surgery. Recent advances in computer vision and } \\
\text { machine learning have ushered a new wave of } \\
\text { technologies to radically improve patient care. With the } \\
\text { Information age, a transformation in workflow and } \\
\text { efficiency similar to that of the Industrial Revolution has }\end{array}$ \\
\hline
\end{tabular}


Younis. Impact of Artificial Intelligence Integration on Surgical Outcome

Table 2: Brief summary of studies included in systematic review

\begin{tabular}{ccccc}
\hline Source & $\begin{array}{c}\text { Year of } \\
\text { Publication }\end{array}$ & Population & Sample Size & Study Design \\
\hline Bagnall et al. [27] & 2018 & $\begin{array}{c}\text { Patients undergoing } \\
\text { colorectal surgery }\end{array}$ & 1380 & Retrospective \\
\hline Adhikari et al. [28] & 2019 & $\begin{array}{c}\text { Patients subjected to } \\
\text { inpatient surgery }\end{array}$ & 2911 & Retrospective \\
\hline Bertsimas et al. [29] & 2018 & $\begin{array}{c}\text { Emergency surgery } \\
\text { patients }\end{array}$ & 382,960 & Retrospective \\
\hline Bihorac et al. [30] & 2019 & $\begin{array}{c}\text { Patients undergoing } \\
\text { major surgery }\end{array}$ & 51,457 & Retrospective \\
\hline Brennan et al. [31] & 2019 & Physicians & 20 & Prospective \\
\hline Koyner et al. [32] & 2018 & Inpatients & 121,158 & Retrospective \\
\hline Delahanty et al. [33] & 2018 & ICU patients & 237,173 & Retrospective \\
\hline Clark et al. [34] & 2018 & Surgical patients & 885,502 & Retrospective \\
\hline Stacey et al. [35] & 2017 & $\begin{array}{c}\text { Randomized trials } \\
\text { about decision aids }\end{array}$ & 105 & Systematic review \\
\hline
\end{tabular}

started, and surgery is expected to benefit from this explosion of information technology. ${ }^{21}$ Therefore, it is essential for surgeons to get a foundational knowledge of $\mathrm{Al}$ and fully comprehend its influence on surgical decision-making.

Pre-operative diagnosis and optimization, in which surgeons prepare a patient for the intended surgical procedure utilizing available imaging and electronic medical records, is important for the accomplishment of any given procedure. Al has been able to assist with efficient cognitive tools to evaluate and stratify patients for risk of complications related to surgery so that such issues can be successfully circumvented. These are appropriately employed in the preoperative clinic and ensure reduction in time delays by making available all relevant reports and safeguard that all risk factors are optimally mitigated so that the anesthesiologist and the surgical team are well equipped with all the vital information they require to safely conduct the surgery. ${ }^{1,22}$

Besides conventional techniques for machine learning and image processing, deep learning-based approaches for preoperative planning are now on the rise. ${ }^{23}$ In the latter case, the network classification architecture consists of convolutional layers for the extraction of information from input data images. Chilamkurthy and colleagues describe using adept deep learning algorithms evaluating $\mathrm{CT}$ images to acknowledge intracranial hemorrhage, skull fractures, intracranial mass effects with shift of the midline. ${ }^{24}$ Though promising human-level precision with good reproducibility is reported ${ }^{25}$, but the feasibility of these methodologies continues to remain a potential barrier to regulatory considerations. One of the examples depict a profoundly stacked convolutional autoencoder instructed to derive biological characteristics to identify prostate cancer utilizing 4D PositronEmission Tomography (PET) pictures. ${ }^{27}$

Gibson used similar technology to segment the abdominal cavity structures from CT images to manage the endoscopic pancreatic and biliary procedures. ${ }^{28} \mathrm{MRI}$ has been combined with user-defined bounding boxes for interactive segmentation of the placenta and fetal brains. $^{29}$

Traditional algorithms typically evaluate iteratively a parametric transformation in order to minimize a given metric between the two medical digital images, volumes or methods. The conventional time consuming and optimization-based registration algorithm has recently been replaced with deep regression models which have vastly improved the time and efficiency for optimizing patients undergoing surgery. ${ }^{30}$

Learning techniques have been thoroughly incorporated into intraoperative guidance production to ensure improved visualization and position in surgery. Al also helps to identify patients at risk of having pain and nausea after surgery, so that a prevention plan may be instituted early on. All the information emanating from the different electronic equipment, and sensors could be combined to establish an algorithm which can make sense of the surgical processes taking place in the operating room in real time and determine the current status of the ongoing surgery. It is further observed that the tendency towards intra-luminal operations and endoscopic surgery is growing, powered by sophisticated 
radiological adjuncts and guided interventions. Techniques armed with navigation assist programs are being researched to direct endoscope maneuvers in order to target specific locations. ${ }^{31-33}$ Mountney et al, ${ }^{34}$ implemented a web-based learning system that can update tracker function by choosing the right characteristics from the categorization of the decision tree. With Minimally Invasive Surgery, surgical robots can achieve superior efficiency with the advancement of $\mathrm{Al}$ techniques. ${ }^{35}$ Its aim is to improve the ability of surgical robotics to interpret the dynamic in vivo environment, make decisions and undertake the prescribed tasks with greater accuracy and productivity. Soft tissue monitoring has also been extended to learning approaches in minimal invasive surgery (MIS).

Al helps to ensure the use of interventions that can maximize recovery and decrease the likelihood of complications based on patient data and help the patient recover much quicker. ${ }^{19}$ Infusion pumpelectronic health record makes sure that a surgeon's order is carried out effectively, thereby reducing the possibility of medical errors post-operatively. Additionally, a system can be installed which gives out a warning that is promptly forwarded to a handheld device used by a nurse. The nurse will assess if an immediate action is needed or can pause it if there is no urgency. Another advantage of post-operative infusion pumps is that the hospital can collect data from the pumps and determine optimal management strategy of the infusion therapy. In this way Al can be used to optimize performance and minimize overall costs and complications. ${ }^{19}$

Al outperforms the judgment of clinicians when triaging postoperative patients for critical care. A machine-learned algorithm demonstrates promise as an everyday tool to aid surgeons chooses the best level of care for their patients immediately after surgeries. It can triage patient's symptoms and advise so that they can determine whether to go to the emergency room or seek treatment in a different setting. ${ }^{20}$

Surgeons, as the primary players in implementing Albased surgical care innovations, will explore opportunities to work with data scientists to capture new types of clinical data and help create concrete interpretations of that data. ${ }^{36,37}$ Surgeons have the clinical experience that can direct data scientists and engineers with the right data to address the right questions, whereas engineers could provide automated, analytical solutions to problems with data analytics, which would otherwise be too expensive or time-consuming for manual methods. ${ }^{38}$
Ultimately, the surgeons are the ones who provide patients with clinical information and will have to develop a conceptual framework for patient communication to relay the data made available by Al. The understanding of Al would be crucial in simplifying complex analysis of risk forecasts, prognoses, and treatment models so as to streamline treatment modalities for patients within the correct clinical context. ${ }^{39}$

There are certain limitations of this study. Firstly, a selection bias may be elicited due to the retrospective nature of included studies. Secondly, the data used in the review was based on web-based search of databases which may leave a possibility of inclusion of a falsely coded data. Third, limiting data to studies from 2015 onwards may have ruled out very initial research that evaluated the influence of using $\mathrm{Al}$ on surgical decision making. Finally, we excluded studies in which no full text was present, that may have led to some misjudgment in the search period of the range of approaches and external authentication.

\section{CONCLUSION}

The progress in the field of Al has transformed current surgical practice into a more decisive and adept science. Important advances have been made in pre-operative planning, intra-operative instruction, and postoperative treatment by using these techniques. In preoperative phase, Al has been broadly accepted for anatomical classification through image recording for determination of optimal surgical planning. Al approaches have already made a significant contribution to more accurate guidance for MIS. Postoperative data from personal devices is integrated with data from hospital stay to minimize comorbidities after discharge. The integration of Al would enable surgical robotics to develop an understanding of the complex surgical scenarios, make real-time decisions and assist in highly accurate and safe surgical procedures.

Received: July 01, 2020

Accepted: April 27, 2021

\section{REFERENCES}

1- Raymond BL, Wanderer JP, Hawkins AT, Geiger TM, Ehrenfeld JM, Stokes JW, et al. Use of the American College of Surgeons National Surgical Quality Improvement Program Surgical Risk Calculator During Preoperative Risk Discussion: The Patient Perspective. Anesth Analg 2019; 128:643-50.

doi: 10.1213/ANE.0000000000003718 
2- $\quad$ Lubitz AL, Chan E, Zarif D, Ross H, Philp M, Goldberg AJ, et al. American College of Surgeons NSQIP Risk Calculator Accuracy for Emergent and Elective Colorectal Operations. J Am Coll Surg 2017; 225:601-11. doi:10.1016/j.jamcollsurg.2017.07.1069

3- Hyde LZ, Valizadeh N, Al-Mazrou AM, Kiran RP. ACSNSQIP risk calculator predicts cohort but not individual risk of complication following colorectal resection. Am J Surg 2019; 218:131-5.

doi:10.1016/j.amjsurg.2018.11.017

4- $\quad$ Leeds IL, Rosenblum AJ, Wise PE, Watkins AC, Goldblatt $\mathrm{MI}$, Haut ER, et al. Eye of the beholder: Risk calculators and barriers to adoption in surgical trainees. Surgery 2018; 164:1117-23. doi: 10.1016/j.surg.2018.07.002

5- Blumenthal-Barby JS, Krieger H. Cognitive biases and heuristics in medical decision making: a critical review using a systematic search strategy. Med Decis Making 2015;35:539-57. doi:10.1177/0272989X14547740

6- Che Z, Purushotham S, Khemani R, Liu Y. Interpretable Deep Models for ICU Outcome Prediction. AMIA Annu Symp Proc 2017; 2016:371-80.

7- Komorowski M, Celi LA, Badawi O, Gordon AC, Faisal AA. The Artificial Intelligence Clinician learns optimal treatment strategies for sepsis in intensive care. Nat Med 2018;24:1716-20.

doi: $10.1038 / 541591-018-0213-5$

8- Henry KE, Hager DN, Pronovost PJ, Saria S. A targeted real-time early warning score (TREWScore) for septic shock. Sci Transl Med 2015; 7:299ra122. doi:10.1126/scitranslmed.aab3719

9- Hao Du, Ghassemi MM, Mengling Feng. The effects of deep network topology on mortality prediction. Annu Int Conf IEEE Eng Med Biol Soc 2016; 2016:2602-05. doi:10.1109/EMBC.2016.7591263

10- Chilamkurthy S, Ghosh R, Tanamala S, Biviji M, Campeau NG, et al. Deep learning algorithms for detection of critical findings in head CT scans: a retrospective study. Lancet 2018;392:2388-96.

\section{doi: 10.1016/S0140-6736(18)31645-3}

11- Meyer A, Zverinski D, Pfahringer B, Kempfert J, Kuehne $\mathrm{T}$, Sündermann $\mathrm{SH}$, et al. Machine learning for real-time prediction of complications in critical care: a retrospective study. Lancet Respir Med 2018; 6:905-14. doi:10.1016/S2213-2600(18)30300-X

12- Li X, Zhang S, Zhang Q, Wei X, Pan Y, Zhao J, et al. Diagnosis of thyroid cancer using deep convolutional neural network models applied to sonographic images: a retrospective, multicohort, diagnostic study. Lancet Oncol 2019; 20:193-201.

doi: $10.1016 / \mathrm{S} 1470-2045(18) 30762-9$

13- Rubinstein E, Salhov M, Nidam-Leshem M, White V, Golan S, Baniel J, et al. Unsupervised tumor detection in Dynamic PET/CT imaging of the prostate. Med Image Anal 2019; 55:27-40.

doi:10.1016/j.media.2019.04.001

14- Gibson E, Giganti F, Hu Y, Bonmati E, Bandula S, Gurusamy K, et al. Automatic Multi-Organ Segmentation on
Abdominal CT With Dense V-Networks. IEEE Trans Med Imaging 2018; 37:1822-34. doi:10.1109/TMI.2018.2806309

15- Winkels $M$, Cohen TS. Pulmonary nodule detection in CT scans with equivariant CNNs. Med Image Anal 2019; 55:15-26. doi:10.1016/j.media.2019.03.010

16- Cool D, Downey D, Izawa J, Chin J, Fenster A. 3D prostate model formation from non-parallel 2D ultrasound biopsy images. Med Image Anal 2006; 10:875-87. doi:10.1016/j.media.2006.09.001

17- Toth D, Pfister M, Maier A, Kowarschik M, Hornegger J. (2015) Adaption of 3D Models to 2D X-Ray Images during Endovascular Abdominal Aneurysm Repair. Medical Image Computing and Computer-Assisted Intervention. Springer Cham 2015; 9349. 339-46. doi.org/10.1007/978-3-319-24553-9_42

18- Liu F, Zhou Z, Samsonov A, Blankenbaker D, Larison W, Kanarek A, et al. Deep Learning Approach for Evaluating Knee MR Images: Achieving High Diagnostic Performance for Cartilage Lesion Detection. Radiology 2018;289:160-9.

doi: 10.1148/radiol.2018172986

19- Gijsberts CM, Groenewegen KA, Hoefer IE, Eijkemans $\mathrm{MJ}$, Asselbergs FW, Anderson TJ, et al. Race/Ethnic Differences in the Associations of the Framingham Risk Factors with Carotid IMT and Cardiovascular Events. PLoS One 2015; 10:e0132321.

doi: 10.1371/journal.pone.0132321

20- Mountney P, Stoyanov D, Davison A, Yang GZ. Simultaneous stereoscope localization and soft-tissue mapping for minimal invasive surgery. Med Image Comput Comput Assist Interv 2006; 9:347-54.

doi: $10.1007 / 11866565 \_43$

21- Topol EJ. High - performance medicine: the convergence of human and artificial intelligence. Nat Med 2019; 25:44-56.

\section{doi:10.1038/s41591-018-0300-7}

22- Mirnezami R, Ahmed A. Surgery 3.0, artificial intelligence and the next-generation surgeon. Br J Surg 2018; 105:463-5.doi: $10.1002 /$ bjs.10860

23- Bouget D, Benenson R, Omran M, Riffaud L, Schiele B, Jannin P. Detecting Surgical Tools by Modelling Local Appearance and Global Shape. IEEE Trans Med Imaging 2015;34:2603-17.

doi: 10.1109/TMI.2015.2450831

24- Shvets AA, Rakhlin A, Kalinin AA, Iglovikov VI. Automatic instrument segmentation in robot-assisted surgery using deep learning. In2018 17th IEEE International Conference on Machine Learning and Applications (ICMLA) 2018; 624-8.

25- Islam M, Atputharuban DA, Ramesh R, Ren H. Real-time instrument segmentation in robotic surgery using auxiliary supervised deep adversarial learning. IEEE Robotics and Automation Letters 2019; 4:2188-95.

26- Liberati A, Altman DG, Tetzlaff J, Mulrow C, Gotzsche PC, loannidis JP, et al. The PRISMA statement for reporting systematic reviews and meta-analyses of 
studies that evaluate health care interventions: explanation and elaboration. PLoS Med 2009; 6:e1000100. doi:10.1371/journal.pmed.1000100

27- Bagnall NM, Pring ET, Malietzis G, Athanasiou T, Faiz $\mathrm{OD}$, Kennedy $\mathrm{RH}$, et al. Perioperative risk prediction in the era of enhanced recovery: a comparison of POSSUM, ACPGBI, and E-PASS scoring systems in major surgical procedures of the colorectal surgeon. Int J Colorectal Dis 2018; 33:1627-34.

doi: $10.1007 / 500384-018-3141-4$

28- Adhikari L, Ozrazgat-Baslanti T, Ruppert M, Madushani RW, Paliwal S, Hashemighouchani $\mathrm{H}$, et al. Improved predictive models for acute kidney injury with IDEA: Intraoperative Data Embedded Analytics. PLoS One 2019; 14:e0214904.

doi: 10.1371/journal.pone.0214904

29- Bertsimas D, Dunn J, Velmahos GC, Kaafarani HMA. Surgical Risk Is Not Linear: Derivation and Validation of a Novel, User-friendly, and Machine-learning-based Predictive OpTimal Trees in Emergency Surgery Risk (POTTER) Calculator. Ann Surg 2018; 268:574-83. doi: $10.1097 / S L A .0000000000002956$

30- Bihorac A, Ozrazgat-Baslanti T, Ebadi A, Motaei A, Madkour M, Pardalos PM, et al. MySurgeryRisk: Development and Validation of a Machine-learning Risk Algorithm for Major Complications and Death After Surgery. Ann Surg 2019; 269:652-62. doi: 10.1097/SLA.0000000000002706

31- Brennan M, Puri S, Ozrazgat-Baslanti T, Feng Z, Ruppert $M$, Hashemighouchani $\mathrm{H}$, et al. Comparing clinical judgment with the MySurgeryRisk algorithm for preoperative risk assessment: A pilot usability study. Surgery 2019; 165:1035-45.

doi:10.1016/j.surg.2019.01.002
32- Koyner JL, Carey KA, Edelson DP, Churpek MM. The Development of a Machine Learning Inpatient Acute Kidney Injury Prediction Model. Crit Care Med 2018;46:1070-7.doi:10.1097/CCM.0000000000003123

33- Delahanty RJ, Kaufman D, Jones SS. Development and Evaluation of an Automated Machine Learning Algorithm for In-Hospital Mortality Risk Adjustment Among Critical Care Patients. Crit Care Med 2018; 46:e481-e488. doi:10.1097/CCM.0000000000003011

34- Clark DE, Fitzgerald TL, Dibbins AW. Procedure-based postoperative risk prediction using NSQIP data. J Surg Res 2018;221:322-7. doi:10.1016/j.jss.2017.09.003

35- Stacey D, Legare F, Lewis K, Barry MJ, Bennett CL, Eden $K B$, et al. Decision aids for people facing health treatment or screening decisions. Cochrane Database Syst Rev 2017; 4:CDo01431. doi: 10.1002/14651858.CDo01431

36- Knops AM, Legemate DA, Goossens A, Bossuyt PM, Ubbink DT. Decision aids for patients facing a surgical treatment decision: a systematic review and metaanalysis. Ann Surg 2013; 257: 860-6.

doi:10.1097/SLA.ob013e3182864fd6

37- Hashimoto DA, Rosman G, Rus D, Meireles OR. Artificial Intelligence in Surgery: Promises and Perils. Ann Surg 2018; 268:70-6.doi:10.1097/SLA.0000000000002693

38- Twinanda AP, Shehata S, Mutter D, Marescaux J, de Mathelin M, Padoy N. EndoNet: A Deep Architecture for Recognition Tasks on Laparoscopic Videos. IEEE Trans Med Imaging 2017; 36:86-97.

doi:10.1109/TMI.2016.2593957

39- Kendale S, Kulkarni P, Rosenberg AD, Wang J. Supervised Machine-learning Predictive Analytics for Prediction of Postinduction Hypotension. Anesthesiology 2018;129:675-8.

doi: $10.1097 / A L N .0000000000002374$ 